\title{
In Vivo Microdialysis to Study Striatal Dopaminergic Neurodegeneration
}

\author{
Giuseppe Di Giovanni, Massimo Pierucci, Mauro Pessia, \\ and Vincenzo Di Matteo
}

\begin{abstract}
Microdialysis cerebral technique has been widely employed in order to study neurotransmitter release. This technique presents numerous advantages such as it allows work with sample in vivo from freely moving animals. Different drugs in different points implanted probes in several brain areas can be infused simultaneously by means of microdialysis. Parkinson's disease (PD) is a progressive neurodegenerative disorder that is primarily characterized by the degeneration of dopamine (DA) neurons in the nigrostriatal system, which in turn produces profound neurochemical changes within the basal ganglia, representing the neural substrate for Parkinsonian motor symptoms. Over the years, a broad variety of experimental models of the disease have been developed and applied in diverse animal species. The two most common toxin models used employ 6-hydroxydopamine (6-OHDA) and the 1-methyl-4-phenyl-1,2,3,6-tetrahydropyridine/1methyl-4-phenilpyridinium ion $\left(\mathrm{MPTP} / \mathrm{MPP}^{+}\right)$, either given systemically or locally applied into the nigrostriatal pathway, to resemble PD features in animals. Both neurotoxins selectively and rapidly destroy catecholaminergic neurons, although with different mechanisms. Since in vivo microdialysis coupled to high-performance liquid chromatography (HPLC) is an established technique for studying physiological, pharmacological, and pathological changes of a wide range of low molecular weight substances in the brain extracellular fluid, here we describe a rapid and simple microdialysis technique that allows the direct quantitative study of the damage produced by $6-\mathrm{OHDA}$ and $\mathrm{MPP}^{+}$toxins on dopaminergic (DAergic) striatal terminals of rat brain.
\end{abstract}

Key words: Parkinson's disease, In vivo microdialysis, Corpus striatum, $\mathrm{MPP}^{+}, 6-\mathrm{OHDA}$, ROS

\section{Introduction}

Parkinson's disease (PD) is a progressive neurodegenerative disorder that is primarily characterized by the degeneration of dopamine (DA) neurons in the nigrostriatal system, which in turn produces profound neurochemical changes within the basal ganglia (Fig. 1), representing the neural substrate for Parkinsonian motor 

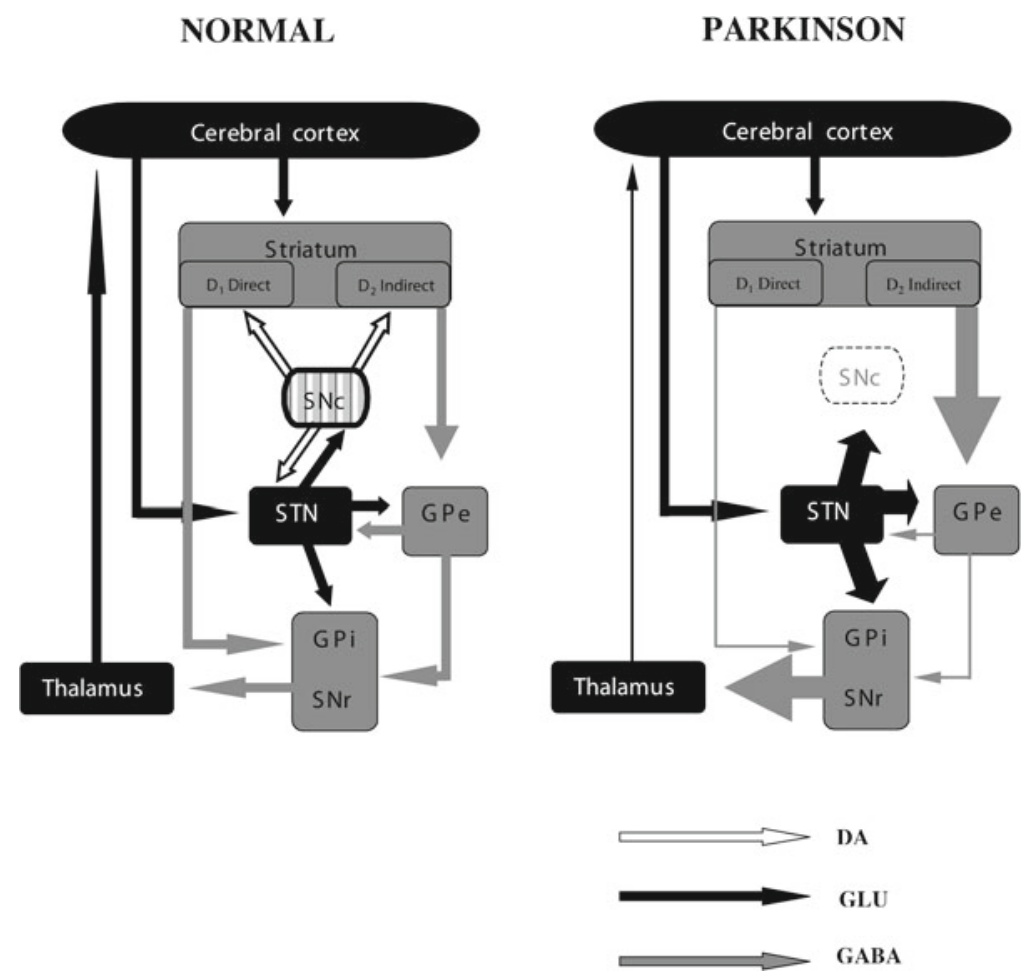

Fig. 1. Simplified diagram illustrating the changes occurring in the basal ganglia functional organization in Parkinson's disease, with respect to normal condition. Relative thickness of arrows indicate the degrees of activation of the transmitter pathways. The basal ganglia participate in larger circuits that also include cortex and thalamus. The striatum is the principal input structure of the basal ganglia, and the internal segment of the globus pallidus (GPi) and the substantia nigra pars reticulata (SNr) are the major output structures, projecting toward the thalamus and brainstem. According to conventional anatomical models, basal ganglia input and output structures are linked via a monosynaptic "direct" pathway and a polysynaptic "indirect" pathway that involves the external pallidal segment (GPe) and the subthalamic nucleus (STN). Dopamine released from terminals of the nigrostriatal $(\mathrm{SNc})$ projection is thought to modulate basal ganglia activity by inhibiting activity along the "indirect" pathway through stimulation of dopamine D2 receptors and enhancing activity along the "direct" pathway by the stimulation of the dopamine D1 receptor. The same model has been applied to explain aspects of the pathophysiology of parkinsonism. Loss of striatal dopamine is believed to result in increased striatal inhibition of GPe, leading to disinhibition of STN neurons and to increased basal ganglia output from GPi and SNr. Increased and altered basal ganglia output to the thalamus is thought to disturb cortical processing, which is ultimately responsible for the development of many of the Parkinsonian motor signs. Dopamine (DA) unfilled arrows, glutamate (GLU) black arrows, $\gamma$-aminobutyric acid (GABA) gray arrows.

symptoms (1). The pathogenesis of the disease is still not completely understood, but environmental and genetic factors are thought to play relevant roles. Important factors include formation of free radicals, impaired mitochondrial activity, increased sensitivity to apoptosis, excitoxicity, and inflammation $(1,2)$. It appears clear that understanding the etiopathogenesis of $\mathrm{PD}$, the modalities 
whereby the neurodegenerative process begins and progresses, is fundamental for the development of drugs to slow or prevent the progression of PD. Most of the progress in this field has been gained thanks to the toxin models of PD. Over the years, a broad variety of experimental models of the disease have been developed and applied in diverse animal species. The two most common toxin models used employ the 6-hydroxydopamine (6-OHDA) and the 1-methyl-4-phenyl-1,2,3,6-tetrahydropyridine/1-methyl-4phenilpyridinium ion $\left(\mathrm{MPTP}_{\mathrm{MPP}}\right)$, given either systemically or locally applied into the nigrostriatal pathway, to resemble PD features in animals. Both neurotoxins selectively and rapidly destroy catecolaminergic neurons, though with different mechanisms (3, 4). In this regard, in vivo microdialysis, a well-established method for monitoring the extracellular levels of neurotransmitters in the CNS (5) allows online estimates of neurotransmitters in living animals and is a suitable method for monitoring the extracellular levels of neurotransmitters during local administration of pharmacological agents. Different doses of a drug or a combination of agonists and antagonists can be administered in the same experiment without adding any fluid to extracellular spaces (6). Therefore, researchers have extensively used this method as a tool for the study of pathophysiologic changes in chemical processes of Parkinsonian brain, and particularly, the infusion of drugs (toxins) through the microdialysis cannula (Fig. 2) (retrodialysis), has permitted the development of some interesting animal models of PD as well as contemporary study of the effects of unilateral lesions of nigrostriatal dopamine neurons (7). Here we will describe a rapid and simple microdialysis technique that allows the direct quantitative study of the damage produced by 6-OHDA and/or $\mathrm{MPP}^{+}$toxins on DAergic striatal terminals of rat brain.

This rapid sampling procedure is completed in 2 days: in the first day of the experiment a fixed toxin (e.g., $\mathrm{MPP}^{+}$or 6-OHDA) dissolved in Ringer solution is perfused through the microdialysis probe, in a target area of the striatum for a short time (10-15 min) with a fixed concentration (generally $1-10 \mathrm{mM}$, or more, depending from the used substance), to induce neurodegeneration of the nigrostriatal system. Forty-eight hours after (day 2), the amount of DA released by the perfusion (challenge) of a second dose of $\mathrm{MPP}^{+}$ could be indicative of the damage produced by a previous perfusion of a toxic compound, since it could be proportional to the number of remaining DAergic terminals (Fig. 3) and the decreases in DA overflow reflecting DA nerve terminal degeneration (8). Indeed, the massive DA extracellular output after the first $\mathrm{MPP}^{+}$or 6-OHDA perfusion is an index of DAergic cell disruption $(9,10)$. Thus, this method is suitable to study different drug-induced DAergic toxicity in the nigrostriatal system and also to estimate the quantitative damage induced by these toxins. 


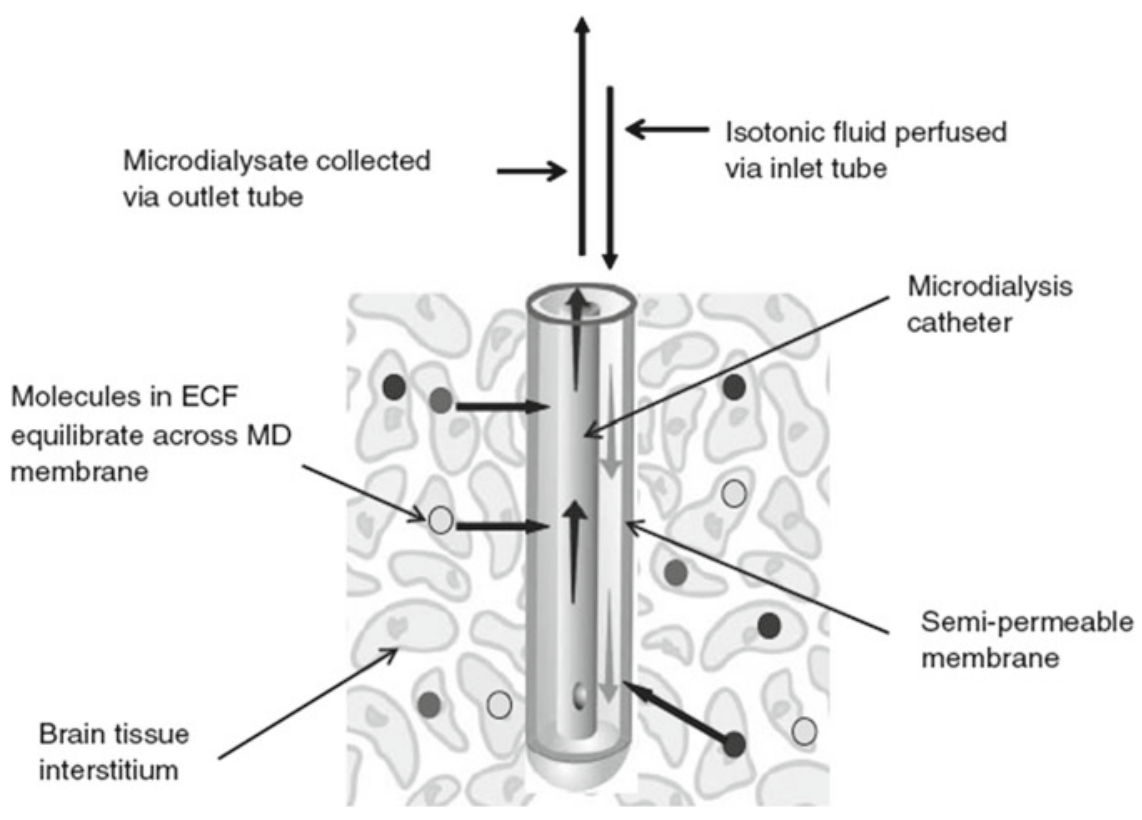

Fig. 2. The Microdialysis probe: A microdialysis (MD) probe is usually constructed as a concentric tube where the perfusion fluid enters through an inner tube, flows to its distal end, exits the tube, and enters the space between the inner tube and the outer dialysis membrane. The direction of flow is now reversed and the fluid moves toward the proximal end of the probe. This is where the "dialysis" takes place, i.e., the diffusion of molecules between the extracellular fluid (ECF) and the perfusion fluid. Vice versa, by reverse dialysis is possible the introduction of a substance into the extracellular space via the microdialysis probe. The inclusion of a higher amount of a drug in the perfusate allows the drug to diffuse through the microdialysis membrane to the tissue. This technique not only allows the local administration of a substance but also permits the simultaneous sampling of the extracellular levels of endogenous compounds.

\subsection{Brief Description of Microdialysis Process}

As already mentioned, microdialysis is a technique designed to monitor the chemistry of extracellular spaces in living tissue and allows monitoring of neurotransmitters released from practically any region of the brain. It consists of the filtration of water-soluble substances in extracellular fluid through a dialysis membrane (Fig. 2) into a perfusion fluid that is collected and then analyzed for the substances of interest. With this technique, extracellular neurotransmitter levels and other molecules equilibrate with the solution flowing through a dialysis probe implanted in discrete brain areas. Usually microdialysis is coupled with high performance liquid chromatography (HPLC), making it possible to detect extracellular levels of many compounds, from small neurotransmitters to larger peptides $(5,6)$. The core of microdialysis is the dialysis probe (Fig. 2) designed to mimic a blood capillary. When a physiological salt solution (artificial cerebral fluid solution, aCSF) is slowly pumped through the microdialysis probe, the solution equilibrates with the surrounding extracellular tissue fluid. After a while, it will then contain a representative proportion of the tissue fluid's molecules, and the microdialysate is extracted and later analyzed in the laboratory, usually by HPLC. The body of any "freely moving" awake microdialysis system consists of a dual-channel microdialysis 

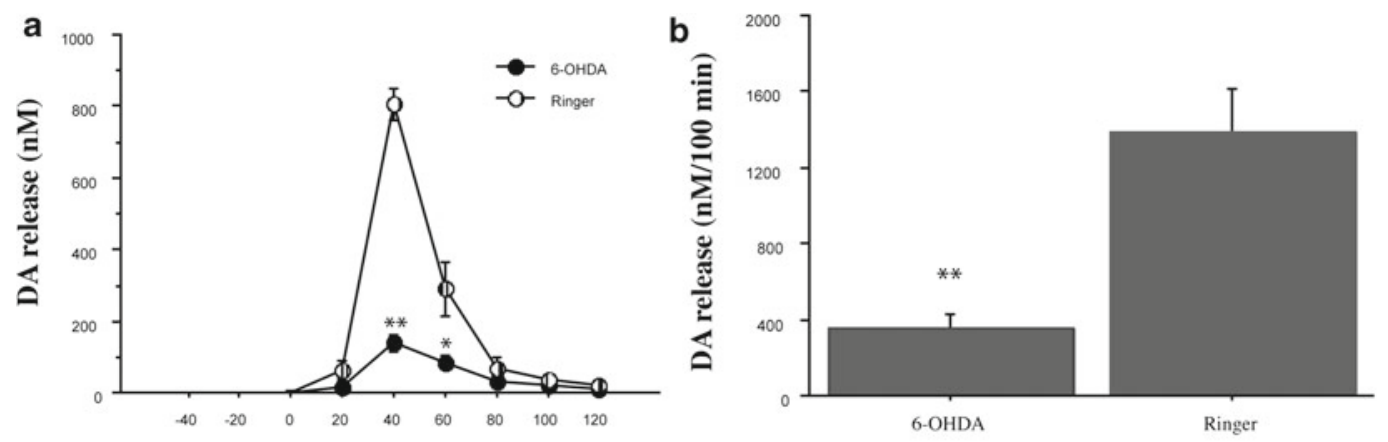

Time (min)
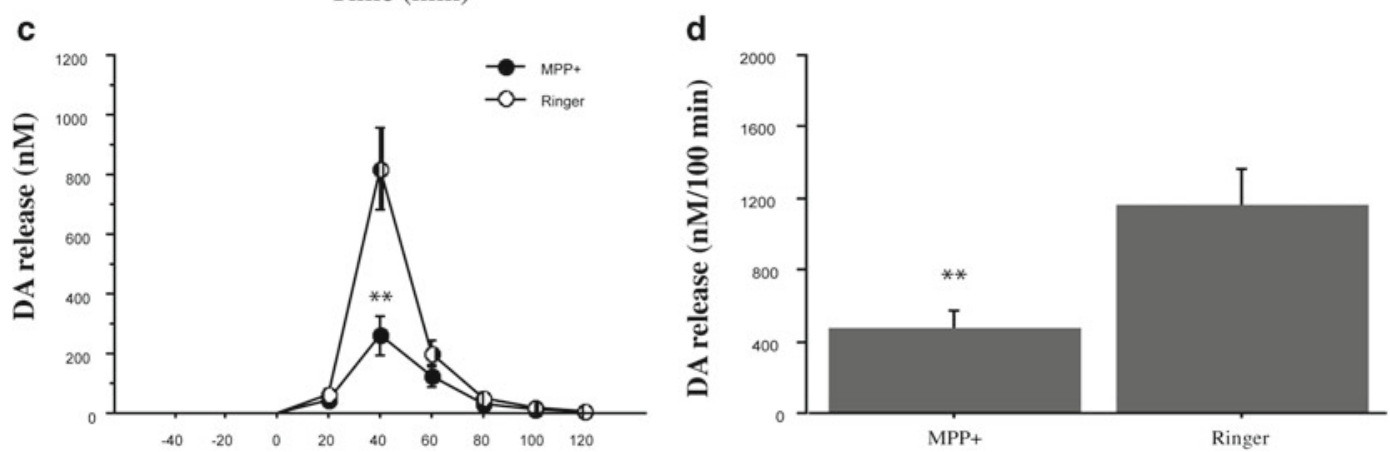

Time (min)

Fig. 3. The 2-day test-challenge microdialysis method. Time course of the effect of $15 \mathrm{~min} 1 \mathrm{mM} \mathrm{MPP}+$ perfusion $24 \mathrm{~h}$ later after perfusion of 6-OHDA (1 mM for $15 \mathrm{~min}$ ) (a), and $1 \mathrm{mM}-15 \mathrm{~min} \mathrm{MPP}^{+}$(c) on extracellular dopamine output in the corpus striatum. Each data point represents mean \pm SEM of absolute levels of DA, without considering probe recovery. Statistical analysis shows that MPP+ and 6-OHDA perfused in the first day of the experiment induced neurodegeneration of nigrostriatal pathway as shown by the decrease in DA release after MPP+ challenge in the second day (one-way ANOVA, followed by Fisher's PLSD post hoc test: ${ }^{\star} p<0.05 ;{ }^{* \star} p<0.016$-0HDA vs. Ringer (Control group), and ${ }^{* \star} p<0.01 \mathrm{MPP}^{+}$vs. Ringer). In (b)-(d) histograms represent the total DA output measured as the sum of five (100 $\mathrm{min})$ consecutive samples after the same conditions of (a) and (c) ${ }^{* \star} p<0.016-0 \mathrm{HDA}$ (b) and MPP+ (d) vs. Ringer). Modified from (13).

swivel that has a quartz-lined center and side channels to minimize dead volume and prevent neurotransmitter oxidation (Fig. 4). Dialysate is typically infused through one channel of the swivel, removed through the other, and then collected with a fraction collector or into a microvial. The head block tether and lever arm are necessary to minimize stress on microdialysis probes. The counterbalanced lever arms generally move vertically and horizontally with the animal to prevent slack in the tether. Most of the lever arms use a mass as the counterbalance, the animal can generally stay in a round polycarbonate container that prevents it from damaging the probes (Fig. 4).

A microdialysis probe is usually constructed as a concentric tube (Fig. 2). The perfusion fluid, delivered by a syringe microdialysis pump (Fig. 4) at low flow rate (perfusion rates of $0.3-3 \mu \mathrm{l} / \mathrm{min}$ are typically used depending upon the volume, sample collection time, and analytical sensitivity needed, with typical sample collection times ranging from 1 to $20 \mathrm{~min}$ ). It enters through an inner tube, 


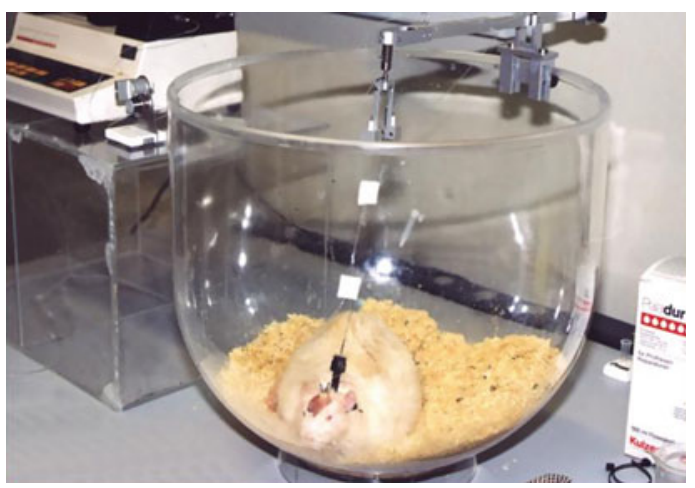

Fig. 4. Photograph of "in vivo freely moving" microdialysis setup. It is formed by a dual-channel microdialysis swivel, head block tether, and lever arm. The aCSF is delivered into the microdialysis probe by a microinjection syringe pump.

flows to its distal end, exits the tube, and enters the space between the inner tube and the outer dialysis membrane, which may be of different lengths, depending on the brain region analyzed. The dialysis membrane is semipermeable and permits free transport of some but not all solutes. Permeability is typically limited to compounds with molecular masses $<20,000 \mathrm{Da}$. The direction of flow is now reversed and the fluid moves toward the near end of the probe (see Fig. 2). Dialysis (the diffusion of molecules between the extracellular and perfusion fluids) takes place at the end of the probe, in the membrane space. It is important to realize that dialysis is an exchange of molecules in both directions. The difference in concentration through the membrane governs the direction of the gradient. It is possible to collect an endogenous compound at the same time that an exogenous compound such as a drug is introduced into the tissue. The gradient of a particular compound into the membrane depends on the difference in concentration between the perfusate and the extracellular fluid and also on the flow velocity inside the microdialysis probe. The absolute recovery (mol/time unit) of a substance from the tissue or of substances entering the tissue from the probe depends on (1) the flow rate of the perfusion fluid, (2) the length of the membrane, (3) the "cut-off" of the dialysis membrane, and (4) the diffusion coefficient of the compound through the extracellular fluid, all important factors to consider for a microdialysis experiment. The advantages and limitations of microdialysis techniques have been reviewed in detail $(5,6,11)$. Moreover, this is an ideal technique because multiple sampling of the brain and several regions in a living free-moving animal is possible. No clean-up procedures such as extraction and homogenization of tissue are required before analysis. Behavioral and pharmacology studies can be contemporaneously performed. Neurochemistry around the brain probes can be continuously monitored. Various drugs can be locally administered in the specific brain areas, without having to inject additional volumes. 


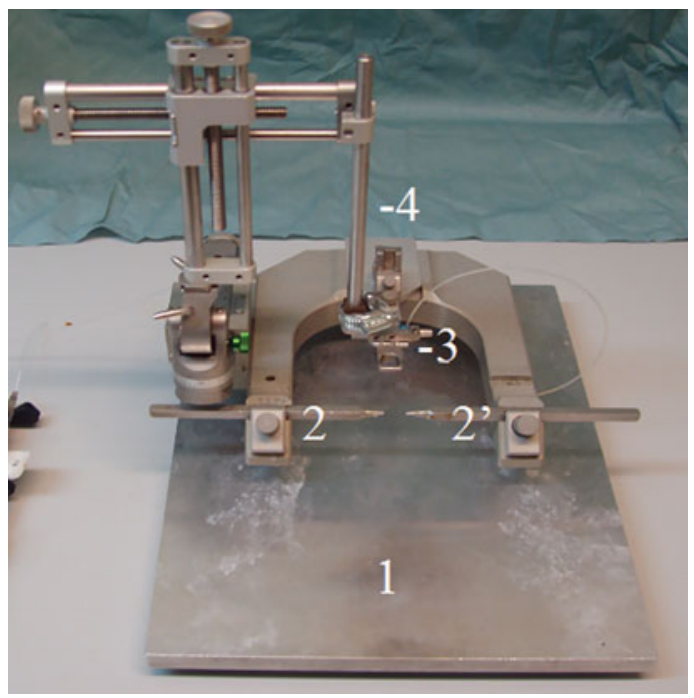

Fig. 5. A typical stereotaxic apparatus: The animal is positioned on the heat-controlled blanket (1), the left (2), and right (2') ear bars and the incisor adaptor with the nose clamp (3) are used to fix the animal's head, and the probe holder (4) is controlled in the $x, y$, and $z$ planes by the stereotaxic arm.

\section{Materials}

\subsection{For Microdialysis}

Male Sprague-Dawley rats (weighing 340-380 g) are obtained from Charles River, Calco, Italy.

Anesthetic: Chloral hydrate.

Stereotaxic atlas for rat (12).

Stereotaxic frame (David Kopf Instruments, Tujunga, USA) (Fig. 5).

Microdialysis guide cannula for CMA/12 probe (Carnegie Medicin, Stockholm, Sweden) (Fig. 4).

Microdialysis probe (CMA/12, 3 mm length, $500 \mu \mathrm{m}$ outer diameter, Carnegie Medicin, Stockholm, Sweden) (Fig. 4).

Scalpel.

Spatula.

Dental or hand drill.

Jeweler's forceps and screwdriver.

3/16 in. bone stainless steel screws.

Methylacrylic cement.

Fluorinated ethylene propylene (FEP) tubing $0.12 \mathrm{~mm}$ inner diameter (Carnegie Medicin, Stockholm, Sweden).

Tubing Adaptors (for connecting FEP Tubing to the probe, swivel, liquid switch, and syringes; Carnegie Medicin, Stockholm, Sweden). 
2.2. Artificial

Cerebrospinal Fluid

\subsection{Drugs}

2.4. For HPLC Analysis
CMA 110 Liquid Switch (Carnegie Medicin, Stockholm, Sweden) (Fig. 4).

Two-channel liquid swivel (Carnegie Medicin, Stockholm, Sweden) (Fig. 4).

CMA/120 system for freely moving animals (Carnegie Medicin, Stockholm, Sweden) (Fig. 4).

Glass Luer-Lock gas-tight microsyringes (Hamilton).

Microperfusion pump (Harvard Apparatus syringe infusion pump 22, USA) (Fig. 4).

Collection plastic vials (e.g., 0.2-ml microcentrifuge tubes) (Fig. 4).

$\mathrm{Na}_{2} \mathrm{HPO}_{4}$.

$\mathrm{NaH}_{2} \mathrm{PO}_{4}$.

$\mathrm{MgCl}_{2}$.

$\mathrm{CaCl}_{2}$.

$\mathrm{KCl}$.

$\mathrm{NaCl}$.

$\mathrm{H}_{2} \mathrm{O}$.

GS $(0.22 \mu \mathrm{m})$ Millipore glass filters.

1-Methyl-4-phenylpiridinium iodide $\left(\mathrm{MPP}^{+}\right)$from Sigma-Aldrich, St. Louis, MO, USA.

6-Hydroxydopamine hydrobromide (6-OHDA) from Aldrich Chem. Co. Milwakee. WI, USA.

HPLC with electrochemical detection (HPLC/EC) system consisting of

HPLC dual-piston pump (LC-10 ADvp pump, Shimadzu Italia, Milano) equipped with pulse dampener.

Manual injector (20- $\mu \mathrm{l}$ sample loop/gas-tight HPLC glass microsyringe).

Coulochem detector (Coulochem II, ESA, Bedford, MA, USA) equipped with a dual electrode analytic cell (model 5014; ESA, Bedford, MA, USA).

Computer software program.

Hypersil column $(\mathrm{Cl} 8,4.6 \times 150 \mathrm{~mm}, 5 \mu \mathrm{m}$, Sigma-Aldrich Chemicals, USA).

$10 \mu \mathrm{M}$ Monoamine standard working solutions (in $0.1 \mathrm{~N}$ percloric acid and $0.05 \% \mathrm{Na}_{2} \mathrm{~S}_{2} \mathrm{O}_{5}$ ) and dilutions (in aCSF) bracketing those estimated for the samples:

4-Hydroxybenzoic acid (4-HBA).

3,4 Dihydroxybenzoic acid (3,4-DHBA).

Dopamine hydrochloride (DA). 
Dihydroxyphenilacetic acid (DOPAC).

HPLC-grade water.

Filtration apparatus consisting of

Borosilicate glass vacuum flask.

Fritted filter support and spring clamp.

$0.22-\mu \mathrm{m}$ Filter discs (e.g., Millipore).

Vacuum source.

Ultrapure helium with regulator and Teflon tubing.

The mobile phase is composed of $70 \mathrm{mM} \mathrm{NaH} \mathrm{PO}_{4}, 0.1 \mathrm{mM}$ $\mathrm{Na}_{2}$ EDTA, $0.7 \mathrm{mM}$ triethylamine, $0.1 \mathrm{mM}$ octylsulfonic acid, and $10 \%$ methanol, adjusted to $\mathrm{pH} 4.8$ with orthophosphoric acid.

Ascorbate.

2.5. Tyrosine Hydroxylase Immuno histochemistry
4\% Paraformaldehyde.

$1 \mathrm{M}$ Phosphate buffer, $\mathrm{pH} 7.4$ (PBS).

Sucrose in PBS,

Isopentane $-45^{\circ} \mathrm{C}$.

Cryostat microtome,

Gelatine-coated slices.

$0.6 \%$ Hydrogen peroxide.

Methanol.

Goat serum.

Humid chamber.

Policlonal rabbit anti- $\beta$ TH (Abcam Limited Cambridge, UK) $0.15 \mu \mathrm{g} / \mu$ l diluted 1:200 in PBS Triton-X-100.

Biotinylated goat anti-rabbit IgG (Vector, 1:200).

Avidin Biotinylated horseradish peroxidase macromolecular Complex solution (Vectastain ${ }^{\circledR}$ ABC system, Vector Labs, Inc., Burlingame, CA).

Standard (Vector) diaminobenzidine/hydrogen peroxidase chromogen (peroxidase substrate kit DAB; Vector Labs, Inc., Burlingame, CA).

\section{Methods} 3.1. Surgery for
Microdialysis
For short- or long-term measurements of neurochemicals in the conscious animal, a guide cannula is typically implanted above the region of interest. This provides support and protection of the more delicate dialysis probe and is a means to introduce the probe without the trauma of a just-completed surgery. Due to trauma resulting from 
the introduction of the probe or the probe/cannula assembly into the tissue, the probe is left in situ for some hours prior to the start of experiments. During this period, the probe is perfused with physiological aCSF.

Male Sprague-Dawley rats (Charles River, Calco, Italy) weighing 340-380 g are used. The animals are kept at constant room temperature $\left(21 \pm 1^{\circ} \mathrm{C}\right)$ and relative humidity $(60 \pm 5 \%)$ under a regular light/dark schedule (light 8.00-20.00 h). Food and water are freely available. Procedures involving animals and their care are conducted in conformity with the institutional guidelines that are in compliance with national (D.L. n. 116, G.U., suppl. 40, 18 Febbraio 1992) and international laws and policies (EEC Council Directive 86/609, OJ L 358,1, Dec. 12, 1987; NIH Guide for the Care and Use of Laboratory Animals, NIH Publication N. 85-23, 1985 and Guidelines for the Use of Animals in Biomedical Research, Thromb. Haemost. 58, 1078-1084, 1987).

1. Prior to surgery, all surgical instruments are autoclaved or sterilized chemically before the probe implantation, a check is made for leaks and air bubbles. A final check is made to ensure that the active length of the probe extends the appropriate distance from the guide cannula tip.

2. The rat is gently restrained and injected intraperitoneally (i.p.) with chloral hydrate $(400 \mathrm{mg} / \mathrm{kg}$, i.p.). Other anesthetics can be used.

3. The animal is mounted in the ear bars of the stereotaxic apparatus (Fig. 5). If the animal is correctly positioned, only movement in the dorsoventral plane should be possible (see Note 1).

4. A midline incision is made using the scalpel blade to pierce the skin.

5. Fascia and connective tissue are scraped away. The entire area is dried with a cotton sponge or Q-tips (see Note 2). The bregma is identified (intersection of the coronal suture with midline) and used to determine the vertical zero coordinate.

6. The guide cannula is attached into the stereotaxic holder and straighten (Fig. 5).

7. The cannula is lowered so that it is centered on the bregma. The anteroposterior (AP) and lateral (L) coordinates are read from the scale and added or subtracted from the stereotaxic coordinates of striatum.

8. By using a 3-Dimensional map of the brain (stereotaxic atlas) along with vernier scales on the apparatus, the guide cannula is placed over the left corpus striatum $(\mathrm{AP}=0.8, \mathrm{~L}=3.0, \mathrm{~V}=-3.0$ from the dura surface and respect to the bregma), according to the atlas of Paxinos and Watson 1986 (12) (see Note 3).

9. Dental or hand drill with a burr sized is appropriately placed for the diameter of the guide cannula or probe assembly on an 
electrode carrier or manipulator that has been modified to accommodate the width of drill. AP and L coordinates may be adjusted. Drill until the dura is punctured, using an up-and-down motion (see Note 4).

10. Three additional holes are drilled around the cannula/probe hole; drill screw holes may be done far enough so as not to interfere with cannula insertion. Using jeweler's forceps and a screwdriver, 3/16-in. bone screws are inserted into the holes (see Note 5 ).

11. The cannula is slowly lowered into the target striatal region until the cannula's base just rests on the dura surface. The ventral (V) coordinate is readed and the cannula lowered to -3.0 from the dura surface. Then, the skull is permanently fixed to with stainless steel screws and methylacrylic cement.

12. The cement is allowed to dry and the holder is carefully removed. The cannula is secured in place by applying additional dental acrylic cement.

13. aCSF is prepared: $147 \mathrm{mM} \mathrm{Na}^{+}, 2.7 \mathrm{mM} \mathrm{K} \mathrm{K}^{+}, 1 \mathrm{mM} \mathrm{Mg}^{2+}$, $1.2 \mathrm{mM} \mathrm{Ca}^{2+}, 154.1 \mathrm{mM} \mathrm{Cl}^{-}$, adjusted to $\mathrm{pH} 7.4$ with $2 \mathrm{mM}$ sodium phosphate buffer. The Ringer solution must be filtered through type GS $(0.22 \mu \mathrm{m})$ Millipore glass filters before use (see Note 6).

14. The microsyringe pump is prepared, filled with aCSF, and attached to the pump.

15. Probe is prepared (see Note 7), and connected to the swivel and syringe by FEP tubing and tubing adaptors (Fig. 4).

16. The dummy cannula is removed from rat guide cannula and the probe is slowly inserted into the striatum (see Note 8).

17. After the probe is inserted, the tether is attached to the animal and placed in the testing cage CMA/120 system for freely moving animals (see Note 9). Balance arm and liquid swivel are connected via a wire guide and spring clamp to a collar that is inserted over the neck of the rat, prior to insertion of the probe. Probe inflow and outflow tubing are secured to the wire guide, which pulls the liquid swivel/balance arm (Fig. 4).

18. Following probe implantation, the rat is allowed to recovery from the surgery and the tissue from the physical damage rendered by probe implantation. The probe is flushed at $0.3 \mu \mathrm{l} /$ min overnight with aCSF.

3.2. First Day of Microdialysis: Lesion of Nigrostriatal DA System
The experiments are performed $24 \mathrm{~h}$ (day l) after probe implantation, in awake, freely moving animals.

1. The corpus striatum is perfused at a flow rate of $1 \mu \mathrm{l} / \mathrm{min}$, and every 20 min samples of perfusate are collected in plastic vials and immediately assayed by HPLC with electrochemical detection (see below Sect. 3.5) (Fig. 7). 


\subsection{Second Day of Microdialysis}

\subsection{Microdialysis to Study Oxidative Stress}

2. After establishing a steady baseline level in three consecutive samples (control value), $\mathrm{MPP}^{+}$iodide or 6-OHDA (1 mM) (see Note 10), dissolved in aCSF, is perfused for $10 \mathrm{~min}$ through the microdialysis probe in the rat striatum to induce neurodegeneration of the nigrostriatal DAergic system, and then with the normal aCSF for further $2 \mathrm{~h}$ at a flow rate of $1 \mu \mathrm{l} / \mathrm{min}$ (see Note 11 ).

3. Afterwards the probe is flushed at $0.3 \mu \mathrm{l} / \mathrm{min}$ until the next day of the experiment.

It is important to note that any pharmacological treatment to prevent neurodegeneration can be done $1 \mathrm{~h}$ before neurotoxins (prevention studies). Moreover, drugs can be systemically given after the first day of the experiment, to study its possible postlesion therapeutic effects. In this case the evaluation of neuronal integrity experiment (day 2 step) must be done at least after a week (see Note 12).

On the first day of the experiment it is important to verify that the drug treatment does not cause any significant effect on basal DA release in the rat striata, and does not modify the extracellular DA release induced by the neurotoxins, which indicate that it does not affect the DA-uptake mechanism or DA metabolism $(13,14)$.

To check the integrity of the DA system, after establishing a steady baseline level in three consecutive samples (control value), $1 \mathrm{mM}$ $\mathrm{MPP}^{+}(1 \mathrm{ml} / \mathrm{min})$ is perfused for $10 \mathrm{~min} 1$ day after (day 2$)$ the first infusion of $\mathrm{MPP}^{+}$or 6-OHDA. Then the perfusion fluid is switched to normal aCSF for $2 \mathrm{~h}$, until the end of experiment. The challenge with $\mathrm{MPP}^{+}$produces a maximal DA extracellular output $40 \mathrm{~min}$ after its infusion (Fig. 3). In a first experimental group, $\mathrm{MPP}^{+}$perfusion produced a maximal DA increase of $819 \pm 136 \mathrm{nmol}$ in the nonlesioned rats (aCSF), $260 \pm 66$ in the $\mathrm{MPP}^{+}$lesioned rats (Fig. 3c, d). In a second group, $\mathrm{MPP}^{+}$perfusion produced a maximal DA increase of $805 \pm 42 \mathrm{nmol}$ in the non-lesioned (aCSF), $139 \pm 22$ in the 6-OHDA lesioned rats (Fig. 3a, b). The limited rise of DA following the $\mathrm{MPP}^{+}$reperfusion is due to toxins-induced neuronal loss in the lesioned groups. The total amounts of DA, released in $100 \mathrm{~min}$, were reduced in $\mathrm{MPP}^{+}$- or 6-OHDA-lesioned rats, challenged with $\mathrm{MPP}^{+}$on day 2 , as compared to controls $(p<0.01$ vs. controls at Fisher's PLSD test, in both groups) (Fig. 3) (13).

Oxidative stress is defined as an imbalance between the production of reactive oxygen species (ROS) and a biological system's ability to readily detoxify the reactive intermediates and/or easily repair the resulting damage. Increasing evidence suggests that reactive oxygen species are involved in the pathophysiology of PD. The microdialysis technique is suitable for measuring radical formation in the extracellular space in vivo. After probe implantation and obtaining a stable baseline, the probe can be used to simultaneously deliver the stimulating agent (e.g., 6-OHDA or $\mathrm{MPP}^{+}$) 


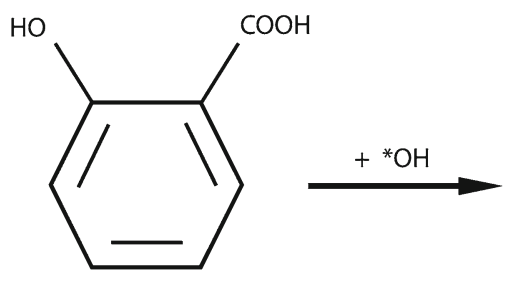

SALICYLIC ACID

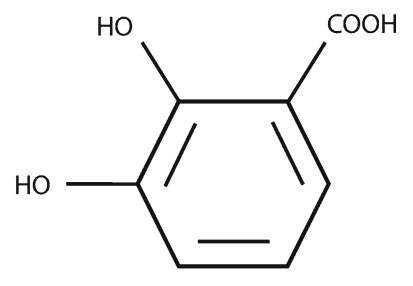

2,3-DHBA

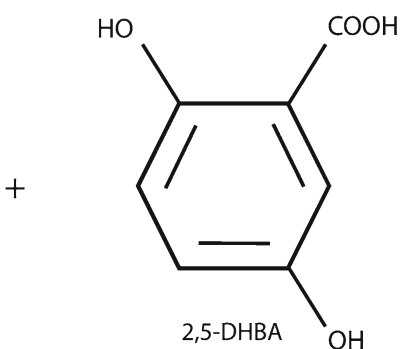

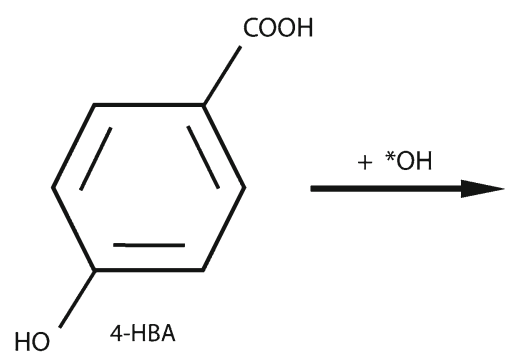

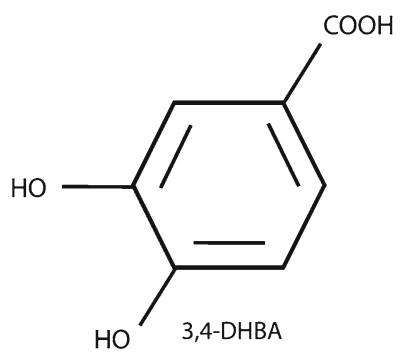

Fig. 6. Reaction scheme of salicylate (2-HBA) with the hydroxyl radicals $\left({ }^{\circ} \mathrm{OH}\right)$ to form 2,3- and 2,5-dihydroxybenzoate (2,3and 2,5-DHBA) and 4-hydroxybenzoic acid (4-HBA) hydroxylation that forms the 3,4-dihydroxybenzoic acid (3,4-DHBA).

and the trapping agent (see below) for the radical determination, without any purification steps before HPLC analysis. In addition, the implanted microdialysis probe allows reliable recording for many hours, and the animal serves as its own control. Due to the high reactivity and short half-life of ROS in biological tissue, indirect methods for in vivo detection have been widely used. The method for in vivo measurement of the hydroxyl radicals $(\cdot \mathrm{OH})$ is based on the ability of the $\mathrm{OH}$ to attack the benzene rings of aromatic molecules (Fig. 6). The salicylate (2-HBA) trapping technique is the most widely used technique for ROS detection in vivo and has previously been employed in experimental animal models of PD with microdialysis and HPLC coupled with an electrochemical detector (ECD) (15-17). However, when ROS react with salicylate two adducts: 2,3- and 2,5-DHBA are formed (Fig. 6). Concerns have been raised with regard to the specificity of the salicylate trapping technique since enzymatic formation of 2,5 -DHBA occurs at extracerebral sites by the cytochrome p450 system in the liver after systemic administration. In addition, 2,3DHBA is metabolized in the rat intestine, making it an unspecific marker (18-20). Salicylate may also chelate divalent metal ions (21), thus affecting hydroxyl radical formation. Finally, salicylate has been shown to affect the inducible nitric oxide synthase (iNOS) (22). Therefore, when a trapping method for ROS detection is employed a very careful evaluation of possible sources of spontaneous ROS formation may be made and these sources may be eliminated as far as possible. Plausible factors affecting hydroxylation 


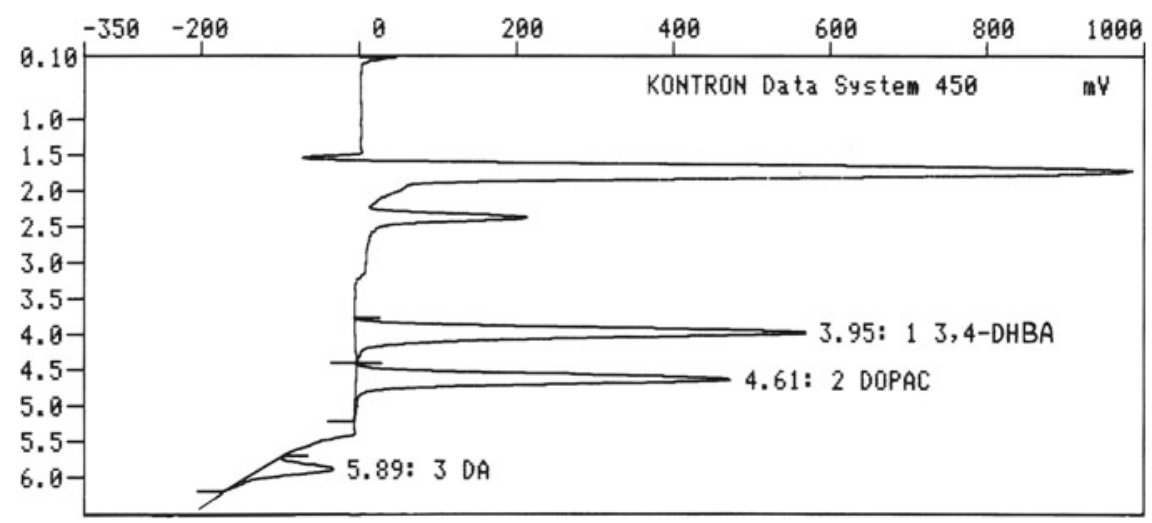

Fig. 7. Chromatogram of 4-hydroxybenzoic acid (4-HBA), dihydroxyphenyl acetic acid (DOPAC), and dopamine (DA).

of DHBAs using microdialysis technique include time, use of metal ions, and reuse of microdialysis probes. The liquid switch perfusion with an artificial physiological solution may possibly corrode some metal surfaces. Reusing the microdialysis probe resulted in increased DHBA production (23). Therefore, to reduce artifactual exogenous hydroxyl free radical production, all stainless steel may be eliminated from the microdialysis setup and HPLC system, the perfusing solutions may kept cold, shielded from the light and freshly prepared just before use. A sensitive and specific hydroxybenzoate (4-hydroxybenzoic acid; 4-HBA) hydroxylation assay is also used in conjunction with HPLC-ECD to monitor hydroxyl radicals $(\cdot \mathrm{OH})$ production $(21,23)$. 4-HBA is known to trap hydroxyl radicals with equal efficacy as salicylate (21). It offers a number of advantages compared to the use of salycilate: firstly, only one isomer is formed by hydroxylation of 4-HBA, the 3,4DHBA (Fig. 7), therefore the signal due to $\mathrm{OH}$ attack is not split between two compounds (2,3- and 2,5-DHBA), decreasing the sensitivity of the assay, as in the case of salycilate (Fig. 7). Secondly, the systemic production of 3,4-DHBA following administration of a large bolus of 4-HBA in vivo is negligible compared to the in vivo enzymatic hydroxylation of salycilate. Lastly, salicylate is an effective chelator of divalent metal ions, including $\mathrm{Fe}^{2+}$. As $\mathrm{Fe}^{2+}$ catalyzes the decomposition of $\mathrm{H}_{2} \mathrm{O}_{2}$ to $\mathrm{OH}$, this may result in an over estimation of apparent $\mathrm{OH}$ production due to its increased local formation $(21,23)$. On the other hand, the formation of 3,4-DHBA may reflect either the production of hydroxyl radicals or other highly reactive species such as peroxynitrite produced by the reaction between superoxide anions and nitric oxide (24). Thus, the formation of 3,4-DHBA is likely to reflect ROS production but is not specific for any single radical species.

We here describe the hydroxybenzoate (4-hydroxybenzoic acid) hydroxylation assay, for the above reasons. 
1. Starting from the day after the surgery, $0.5 \mathrm{mmol} / \mathrm{l}$ 4-hydroxybenzoic acid is added to aCSF solution and perfused continuously.

2. Brain dialysates are collected every $20 \mathrm{~min}$, and immediately assayed for determination of 3,4-dihydroxybenzoic acid $(3,4$ DHBA) (see below Sect. 3.5) (Fig. 7).

3. After a stable baseline, to induce exogenous hydroxyl radicals stimulation, the perfusion fluid is switched for $10 \mathrm{~min}$ to a fluid containing $1 \mathrm{mM} \mathrm{MPP}^{+}$or 6-OHDA (or other toxins able to induce oxidative stress).

4. Then the perfusion fluid is switched back to the previous perfusion fluid (normal aCSF+4-HBA), for further $2 \mathrm{~h}$.

5. In control experiments, only aCSF with 4 - $\mathrm{HBA}$ is used.

3.5. HPLC Analysis

3.6. Immunohistochemistry of Tyrosine Hydroxylase
Dialysate samples are analyzed by reversed-phase HPLC coupled with electrochemical detection (Fig. 7) $(13,14)$.

1. $2 \mathrm{~L}$ of mobile phase composed of $70 \mathrm{mM} \mathrm{NaH}_{2} \mathrm{PO}_{4}, 0.1 \mathrm{mM}$ $\mathrm{Na}_{2}$ EDTA, $0.7 \mathrm{mM}$ triethylamine, $0.1 \mathrm{mM}$ octylsulfonic acid, and $10 \%$ methanol, adjusted to $\mathrm{pH} 4.8$ with orthophosphoric acid (see Note 13) are prepared.

2. The mobile phase solution is filtered through $0.22-\mu \mathrm{m}$ filter under vacuum, then degassed by passing a gentle stream of ultrapure helium through the solution for $10 \mathrm{~min}$ at a pressure of 5-10 psi, using Teflon tubing (see Note 14).

3. Any air bubbles are purged from the mobile phase inlet lines. The HPLC/EC system is assembled connecting the mobile phase to the inlet lines. The flow rate is setted at $1 \mathrm{ml} / \mathrm{min}$ and it is allowed several hours (e.g., 14-16 h) for equilibration of the mobile phase and column.

4. Cell potential of the first electrode is adjusted at $-175 \mathrm{mV}$ and the second at $+175 \mathrm{mV}$, respectively.

5. The electrode sensitivity is adjusted to $500 \mu \mathrm{A}$ for DOPAC and to $5 \mathrm{nA}$ for DA (see Note 15).

6. $20 \mu \mathrm{l}$ of 10-1 nM or less of DA and DOPAC standard working solution are injected to determine retention times and standard curves, used for the linear regression to determine analyte concentration (see Note 16).

7. A linear regression analysis of the standards is performed and the resulting linear regression equation is used to determine actual concentrations in samples.

At the end of the experiments, the rats are perfused transcardially under deep anesthesia (chloral hydrate) with physiological saline (180-200 ml), followed by $4 \%$ paraformaldehyde in phosphate buffer, pH $7.4(180-200 \mathrm{ml})$. 

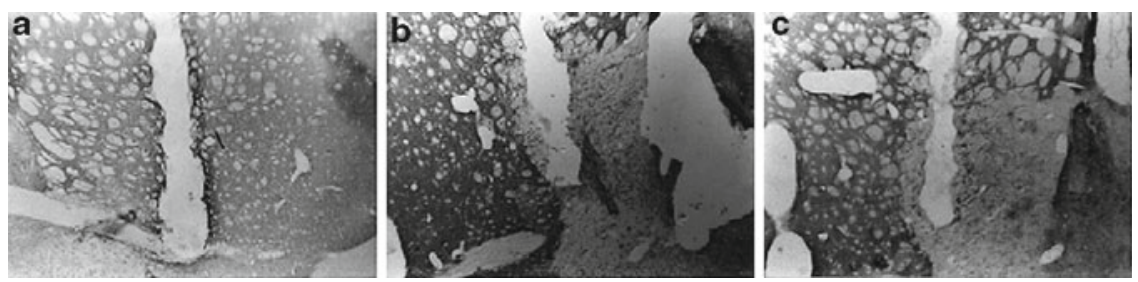

Fig. 8. Photomicrograph of coronal sections (cryostat-cut sections) through striatum (around the microdialysis probe's membrane) after the immunostaining. (a) Rat perfused with Ringer's solution. (b) Rat perfused with MPP+ . (c) Rat perfused with 6-OHDA (magnification 32×). Adapted from (13).

Brains are removed and cryoprotected by serial passages in sucrose in PBS, pH 7.4; first in 10\% sucrose for $24 \mathrm{~h}$ and then in $30 \%$ sucrose for $2-5$ days. The brains are then frozen in isopentane $-45^{\circ} \mathrm{C}$ and then stored at $-80^{\circ} \mathrm{C}$. Coronal sections $(30 \mu \mathrm{m}$ thickness $)$ are cut using a cryostat microtome, mounted in gelatine-coated slices and stained for tyrosine hydroxylase (TH) immunohistochemistry. All experiments are done at room temperature. Sections are washed and then treated with $0.6 \%$ hydrogen peroxide in methanol for $30 \mathrm{~min}$, washed again, and incubated in a solution containing PBS and $50 \%$ goat serum for $30 \mathrm{~min}$ in a humid chamber. Slices are drained and further incubated with policlonal rabbit anti- $\beta \mathrm{TH}$ (Abcam Limited Cambridge, UK) $0.15 \mu \mathrm{g} / \mu$ l diluted 1:200 in PBS containing $1.5 \%$ goat serum and $0.1 \%$ Triton-X-100 over night at $4^{\circ} \mathrm{C}$. Sections are then incubated for $2 \mathrm{~h}$ with biotinylated goat antirabbit IgG (Vector, 1:200) followed by a second 1-h incubation with Avidin Biotinylated horseradish peroxidase macromolecular Complex solution (Vectastain ${ }^{\circledR}$ ABC system, Vector). The peroxidase is then visualized with a standard (Vector) diaminobenzidine/ hydrogen peroxidase chromogen reaction for $5 \mathrm{~min}$. The area which shows reduction in $\mathrm{TH}$ immunoreactivity around the cannula is identified under a microscope (Fig. 8) (13).

3.7. Data Analysis

Dopamine and DOPAC content in each sample is expressed as the absolute dialysate levels in $\mathrm{nmol} / \mathrm{l}$ (without considering probe recovery). Data correspond to mean \pm SEM values of absolute DA and DOPAC levels obtained in each experimental group. 3,4DHBA content in each sample is expressed as a percentage of the average baseline level calculated from three fractions collected before neurotoxins infusion. Data are expressed as mean \pm SEM of the percentage obtained in each experimental group. Data are analyzed by one-way analysis of variance (ANOVA) with repeated measures, followed by the Fisher's protected least significance difference post hoc test (Fisher's PLSD) to allow multiple comparisons between groups. All statistical analyses are performed with StatView ${ }^{\mathrm{TM}}$ version 5.0.1 (SAS Institute, Inc., Cary, NC, USA) $(13,14)$. 
1. Stereotaxic surgery requires that the animal be firmly mounted into the stereotaxic instrument. No horizontal movement of the head should occur. The presence of such indicates improper mounting of the animal. Ear bars may require tightening or reinsertion.

2. Dilute hydrogen peroxide can be applied to facilitate drying of the skull and aid visualization of bregma. This step is critical for the proper adherence of dental acrylic to the skull at the end of this surgical procedure.

3. A stereotaxic atlas provides coordinates for the anterior-posterior $(\mathrm{AP})$, lateral $(\mathrm{L})$, and dorsal ventral (DV) planes. Accurate placement depends on adherence to the reference points used for atlas preparation. Depending on the atlas employed, the position of the head on the incisor bar may be either above or below the interaural line. In addition, brain size may vary depending on strain, age, or sex. Pilot studies should be conducted to validate or adjust the coordinates if necessary.

4. The size of the drill bit should be that which will allow lowering of the cannula into the brain without catching the bone. Caution should be exercised in drilling so that the meninges and underlying brain tissue are not damaged.

5. The screws serve to anchor the cannula/probe assembly when dental acrylic is applied. The screws should be rigid but should not extend to the dura.

6. For $500 \mathrm{ml}$ aCSF: $2 \mathrm{mM} \mathrm{Na}_{2} \mathrm{HPO}_{4}(500 \mathrm{ml})$ and $2 \mathrm{mM}$ $\mathrm{NaH}_{2} \mathrm{PO}_{4}(250 \mathrm{ml})$ should be prepared. The aCSF salts are dissolved in about $350-400 \mathrm{ml}$ of $2 \mathrm{mM} \mathrm{Na}_{2} \mathrm{HPO}_{4}$ and adjusted to pH 7.4 with $2 \mathrm{mM} \mathrm{NaH}_{2} \mathrm{PO}_{4}$, then bringed to $500 \mathrm{ml}$ with $\mathrm{H}_{2} \mathrm{O}$ or $2 \mathrm{mM}$ sodium phosphate buffer $\mathrm{pH} 7.4$.

7. Prior to use, any remaining glycerol from dialysis membrane is removed by soaking in $70 \%$ ethanol for $\sim 5 \mathrm{~min}$. The probe is perfused for several minutes with distilled water or perfusion fluid (flow rate $2 \mu \mathrm{l} / \mathrm{min}$ ) and checked for leaks or air bubbles inside the membrane.

8. Probe insertion requires a steady hand since it is easy to damage the delicate membrane. Lack of outflow, after probe insertion, is often due to occlusion of the outflow tubing. This can be overcome by cutting the tubing. If this does not result in flow, the system (i.e., tubing and pump connectors) should be checked for leaks. If flow still does not occur, the probe is probably at fault. If such is the case, the probe should be removed and inspected for leaks or tears. It is possible to reinsert the probe; however, an ample amount of time must then elapse before sampling can begin. 
9. The outflow line must exit the testing cage in such a way that the experimenter can remove the collection vial without disturbing the animal.

10. To ensure maximal reproducibility, $\mathrm{MPP}^{+}$or $6-\mathrm{OHDA}(10 \times)$ dissolved in $0.1 \%$ ascorbate/saline are prepared in advance, filtered through $0.22 \mu \mathrm{m}$ filters, aliquoted, and stored at $-20^{\circ} \mathrm{C}$. Immediately before use, aliquots gently defrosted, are diluted in normal aCSF to the final concentration of $1 \mathrm{mM}$ each and loaded into a designated microdialysis syringe, protected from light.

11. A liquid switch (CMA 110 Liquid Switch) can be used for delivery of different perfusates into the region dialyzed. With practice, this can also be done manually. Each perfusate switch can result in the introduction of air bubbles into the probe (resulting in a decrease in the active membrane area). Therefore, care must be taken when performing this step.

12. Interestingly, this method can be employed also to study neuroregenerative strategies, since Santiago and colleagues (25) investigated whether a short-lasting infusion of $\mathrm{MPP}^{+}$into the SN can be used as a chronic model of parkinsonism, by allowing a 1-month delay after intranigral $\mathrm{MPP}^{+}$administration, and it was shown that infusion of $\mathrm{MPP}^{+}$into the $\mathrm{SN}$ may cause chronic damage to the nigrostriatal pathway. Therefore, using various DAergic toxins (e.g., 6-OHDA in $\mathrm{SNc}$ ) and DA releasing agents such as high $\mathrm{K}^{+}$or amphetamine, besides $\mathrm{MPP}^{+}$, for the test challenge of the second day or after $1-2$ weeks after the lesion, it is possible to employ this method for both the study of neuroprotective or restorative treatments for PD.

13. Typically, the mobile phase is recycled and replaced one to two times a week. If background noise is detected, however, the recycled mobile phase should be degassed once again. Mobile phase composition is critical for HPLC/EC detection of dopamine and its metabolites. Retention times are affected by alterations in room temperature as well as by $\mathrm{pH}$, the concentration of ion-pairing reagent, and the inorganic solvent employed. These can be varied to modify analyte resolution. Increasing $\mathrm{pH}$ decreases the retention time of acidic metabolites (e.g., DOPAC and HVA). Increasing the concentration of ion-pairing reagents (e.g., octanesulfonate, octylsulfate, pentanesulfonate, or hexanesulfonate) delays retention of amines, enabling better separation from charged molecules. Increasing solvent concentration results in a decreased retention time of amines and their metabolites. Each of these parameters can be modified to increase analyte resolution.

14. Filtration and complete degassing is essential for sensitive electrochemical detection of catecholamines. Particulate matter in the mobile phase can clog the HPLC/EC detector system, creating high pressure and baseline disturbances. Air bubbles 
from improper degassing are the most common problem in EC detection and can wreak havoc on an HPLC/EC system.

15. Under these conditions, the sensitivity for DA is about $0.35 \mathrm{pg} / 20 \mu \mathrm{l}$ with a signal to noise ratio of $3: 1$.

16. Samples are injected manually into the HPLC and detection of DA, DOPAC, or 3,4-DHBA is carried out with a coulometric detector (Coulochem II, ESA, Bedford, MA, USA) coupled to a dual electrode analytic cell (model 5014) (Fig. 7).

\title{
Acknowledgments
}

\begin{abstract}
Authors are indebted to the EU COST Action CMl103 "Structure-based drug design for diagnosis and treatment of neurological diseases: dissecting and modulating complex function in the monoaminergic systems of the brain" for supporting our international collaboration.
\end{abstract}

\section{References}

1. Blandini F, Nappi G, Tassorelli C, Martignoni E (2000) Functional changes of the basal ganglia circuitry in Parkinson's disease. Prog Neurobiol 62:63-88

2. Di Giovanni G (2008) Will it ever become possible to prevent dopaminergic neuronal degeneration? CNS Neurol Disord Drug Targets 7:28-44

3. Schober A (2004) Classic toxin-induced animal models of Parkinson's disease: 6-OHDA and MPTP. Cell Tissue Res 318:215-224

4. Beal MF (2001) Experimental models of Parkinson's disease. Nat Rev Neurosci 2: 325-334

5. Ungerstedt U (1991) Microdialysis: principles and applications for studies in animals and man. J Intern Med 230:365-373

6. Hammarlund-Udenaes M (2000) The use of microdialysis in CNS drug delivery studies: pharmacokinetic perspectives and results with analgesics and antiepileptics. Adv Drug Deliv Rev 45:283-294

7. Di Giovanni G, Esposito E, Di Matteo V (2009) In vivo microdialysis in Parkinson's research. J Neural Transm Suppl 73:223-243

8. Giovanni A, Sonsalla PK, Heikkila RE (1994) Studies on species sensitivity to the dopaminergic neurotoxin 1-methyl-4-phenyl-1,2,3,6tetrahydropyridine. 2. Central administration of 1- methyl-4-phenylpyridinium. J Pharmacol Exp Ther 270:1008-1014
9. Rollema H, Kuhr WG, Kranenborg G, De Vries J, Van den Berg C (1988) $\mathrm{MPP}^{+}$-induced efflux of dopamine and lactate from rat striatum have similar time courses as shown by in vivo brain dialysis. J Pharmacol Exp Ther 245:858-866

10. Tobón-Velasco JC, Silva-Adaya D, CarmonaAparicio L, García E, Galván-Arzate S, Santamaría A (2010) Early toxic effect of 6-hydroxydopamine on extracellular concentrations of neurotransmitters in the rat striatum: an in vivo microdialysis study. Neurotoxicology 31:715-723

11. Benveniste H, Huttemeier PC (1990) Microdialysis: theory and application. Prog Neurobiol 35:195-215

12. Paxinos G, Watson C (1986) The rat brain in stereotaxic coordinates. Academic, New York

13. Di Matteo V, Pierucci M, Di Giovanni G, Di Santo A, Poggi A, Benigno A, Esposito E (2006) Aspirin protects striatal dopaminergic neurons from neurotoxin-induced degeneration: an in vivo microdialysis study. Brain Res 1095:167-177

14. Di Matteo V, Benigno A, Pierucci M, Giuliano DA, Crescimanno G, Esposito E, Di Giovanni G (2006) 7-nitroindazole protects striatal dopaminergic neurons against MPP+induced degeneration: an in vivo microdialysis study. Ann N Y Acad Sci 1089:462-471

15. Chiueh CC, Krishna G, Tulsi P, Obata T, Lang K, Huang S-J, Murphy DL (1992) Intracranial 
microdialysis of salicylic acid to detect hydroxyl radical generation through dopamine autooxidation in the caudate nucleus: effects of $\mathrm{MPP}^{+}$. Free Radic Biol Med 13:581-583

16. Teismann P, Schwaninger M, Weih F, Ferger B (2001) Nuclear factor-kappaB activation is not involved in a MPTP model of Parkinson's disease. Neuroreport 12:1049-1053

17. Obata T (2006) Nitric oxide and $\mathrm{MPP}^{+-}$ induced hydroxyl radical generation. J Neural Transm 113:1131-1144

18. Halliwell $B$, Kaur $H$, Ingelman-Sundberg $M$ (1991) Hydroxylation of salicylate as an assay for hydroxyl radicals: a cautionary note. Free Radic Biol Med 10:439-441

19. Halliwell B, Kaur H (1997) Hydroxylation of salicylate and phenylalanine as assays for hydroxyl radicals: a cautionary note visited for the third time. Free Radic Res 27:239-244

20. Montgomery J, Ste-Marie L, Boismenu D, Vachon L (1995) Hydroxylation of aromatic compounds as indices of hydroxyl radical production: a cautionary note revisited. Free Radic Biol Med 19:927-933

21. Ste-Marie L, Boismenu D, Vachon L, Montgomery J (1996) Evaluation of sodium 4-hydroxybenzoate as an hydroxyl radical trap using gas chromatography-mass spectrometry and high-performance liquid chromatography with electochemical detection. Anal Biochem 241:67-74

22. Amin AR, Vyas P, Attur M, LeszczynskaPiziak J, Patel IR, Weissmann G, Abramson SB (1995) The mode of action of aspirin-like drugs: effect on inducible nitric oxide synthase. Proc Natl Acad Sci U S A 92(17):7926-7930

23. Liu M, Liu S, Peterson SL, Miyake M, Liu KJ (2002) On the application of 4-hydroxybenzoic acid as a trapping agent to study hydroxyl radical generation during cerebral ischemia and reperfusion. Mol Cell Biochem 234(235): 379-385

24. Bogdanov MB, Ferrante RJ, Mueller G, Ramos LE, Martinou JC, Beal MF (1999) Oxidative stress is attenuated in mice overexpressing BCL-2. Neurosci Lett 262:33-36

25. Santiago M, Westerink BHC, Rollema H (1991) Responsiveness of striatal dopamine release in awake animals after chronic 1-methyl-4-phenylpyridinium ion-induced lesions of the substantia nigra. J Neurochem 56:1336-1134 